Journal of Computer Science and Technology Studies (JCSTS))

ISSN: $2709-104 \mathrm{X}$

DOI: $10.32996 / j$ csts

Journal Homepage: www.al-kindipublisher.com/index.php/jcsts

\title{
Internet of Everything: A Global Solution to Digital world
}

\author{
Ebole Alpha Friday ${ }^{1} \square$ Adewale Shomope $^{2}$ and Adeyemo Gbadebo Adebowale ${ }^{3}$ \\ ${ }^{12}$ Department of Computer Science, School of Technology, Lagos State Polytechnic, Nigeria \\ ${ }^{3}$ Department of Computer Science, School of Technology, Grace Polytechnic, Nigeria
}

$\square$ Corresponding Author: Ebole Alpha Friday, E-mail: alphaechoeb@gmail.com

\section{ARTICLE INFORMATION \\ Received: 08 September 2021 \\ Accepted: 14 October 2021 \\ Published: 22 October 2021 \\ DOI: $10.32996 /$ jcsts.2021.3.2.4}

\section{KEYWORDS}

Internet of everything, digital

security, internet of things

\section{ABSTRACT}

The trend of the digital world is the transition from machine-to-machine, machine-topeople, and information technology to human technology with expanded digital to the maturity stage of the internet of everything. The (loE) is the addition of connectivity and intelligence to every device in order to give them special functions. It embraces four components, namely, people, process, data, and things, and also four technical points of view which are smartness, interconnectivity, big data, and semantic interoperability to deploy the solutions that offer programmability, improved flexibility, and enhanced policy management by the various private and public sectors. The architecture of IoE includes applications, service platform, Internet, gateways, communications, and nodes and attract a security need that will be ubiquitous and able to protect the devices, applications, networks, data, users, and things that make up the Internet of Everything. These systems work collaboratively and smartly with each other and perform the desired task. loE will be a radical shift to think on how we live, solve the problem, create value, secure environment to keep people, data, processes, and things under digital hamlet. The network capabilities will create new experiences, capabilities, and economic opportunities for individuals, businesses, and governments as a solution to the digital world. The internet of everything is a philosophy of one thing, which is everything.

\section{Introduction}

The architectural design of the Internet of Everything (IOE) is built on the Internet of Things (IOT) and is defined as a "worldwide network of interconnected objects that are uniquely addressable based on the standard protocols of communication. It is governed by three important paradigms such as internet-oriented (middleware), things-oriented (sensors), and semantic-oriented (knowledge). According to Cluster of European research projects on the Internet of Things - "Things are active participants in business, information and social processes where they are enabled to interact and communicate among themselves and with the environment by exchanging data and information sensed about the environment while reacting autonomously to the real/physical world events and influencing it by running processes that trigger actions and create services with or without direct human intervention".

However, loT goes beyond the mobile computing environment and progresses into linking everyday existing things and embedding intelligence into them. loT focuses on sharing the understanding of their appliances, ubiquitous communication networks and software architectures in the dissemination of information to where it is relevant and analytic tools that aim for smart behaviour and independence. The key technological developments application is shown in the figure below

Copyright: (c) 2021 the Author(s). This article is an open access article distributed under the terms and conditions of the Creative Commons Attribution (CC-BY) 4.0 license (https://creativecommons.org/licenses/by/4.0/). Published by Al-Kindi Centre for Research and Development, London, United Kingdom. 


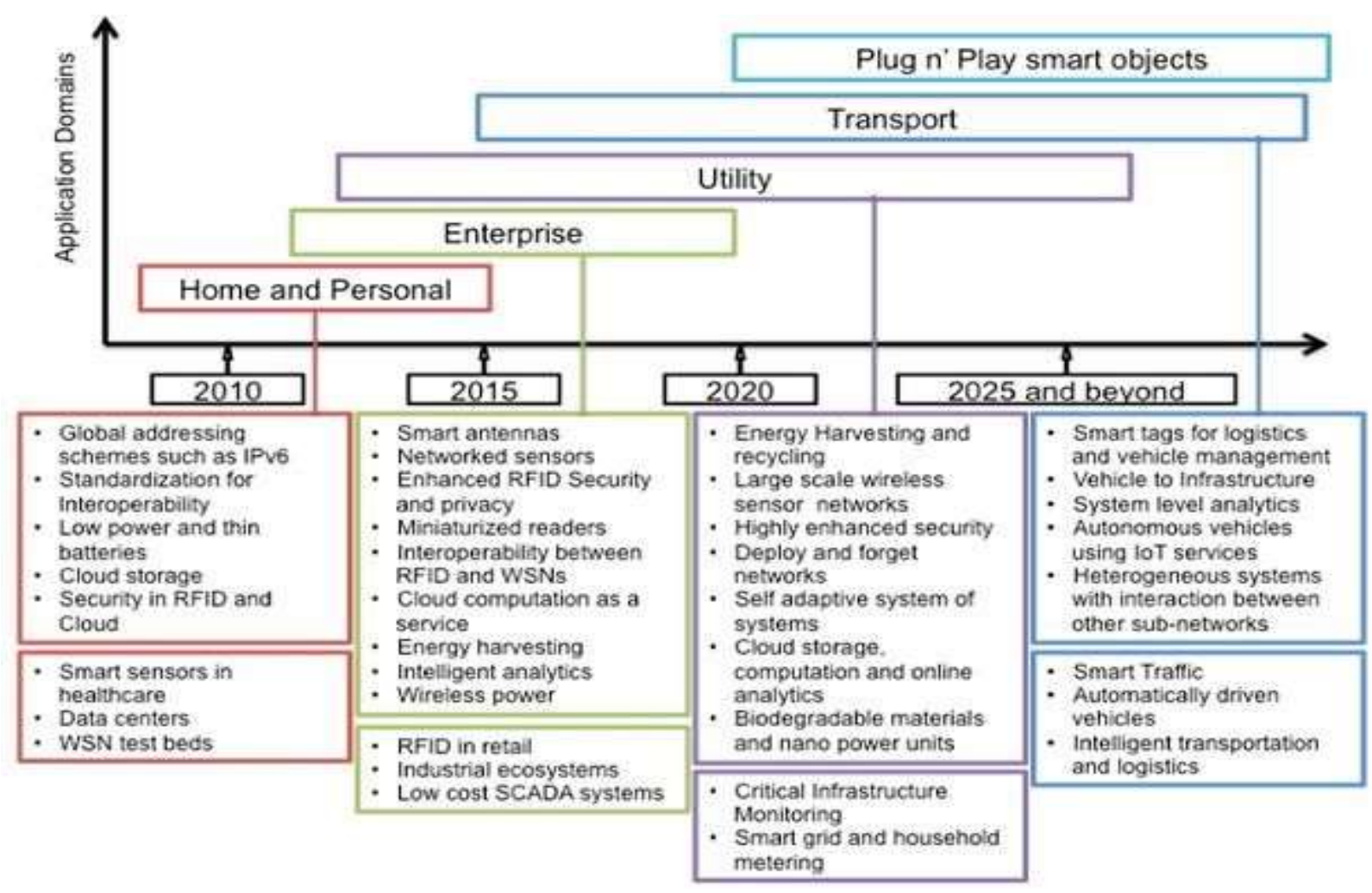

Figure 1: Key technological developments in the context of IoT application domains

Internet of Everything intends to the trend of devices that are linked with the expansion of digital features and regulated through Internet connectivity. loE generates a cyber-physical creation around us as a hamlet for digital devices and consumer products. This approach has necessitated the way we live, work and play and also transform the physiognomies and techniques of our homes, cars, hospitals, factories, businesses etc.

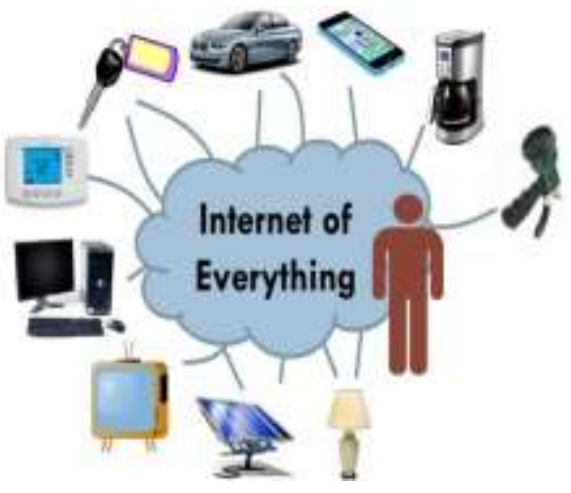

Figure 2. Internet of Everything (IoE)

The loE technology was based on the architectural framework of the Internet of Things (IoT). It was the modification of loT by accumulating more topographies devices that allow the structures to congregate and be accessible to multiple access points. These devices are nurtured to have an intelligent, reliable and secure global network to meet a specific objective and satisfies the purpose by which it was designed. Therefore, promoting an organization on how values are delivered in terms of technology, people-to-machine, machine-to-machine as well as business strategies arrangement. It is a technological solution governing, modernisation, public enhancement, cost optimization, growth, safety and worldwide resource management for social and economic sectors through the availability of financial services, public safety, healthcare, transportation, smartphones, locomotive, effectiveness, dynamism, surveillance, security, financial services, public safety, healthcare, retail, warehouse, industrial, distribution and many more.

Internet of Everything (loE) is a unified interconnection and independent harmonization of enormous integer of computing devices, data, people, processes, workstations, sensors, entities, and machines through a backbone connective of the Internet. 
The awareness of our rationale rests on how will develop our fundamental activities on work, utilization of the machine, human realisation, work collaborative with people and/or machines and unprecedented environment which was not put to thought in the past. IoE has led us to novel innovative solutions to overcome the unique connectivity, interoperability, miniaturization and energy-efficiency challenges and stand on the platform that is highly scalable, flexible, secure to manage billions of workstations of all types with the understanding of a highly sophisticated and integrity rules of online communication with strong security on devices.

The main challenges on the loE are security, which extends and covered all expect of devices, people, data, processes and things as the Internet of Everything mean ubiquitous. Therefore, the security solutions must protect the interconnectivity of data, users, devices, applications and things that make up the Internet of Everything and function collaboratively with each other to achieve a specific result and to satisfy a common goal.

\subsection{Motivation}

Internet of Everything (IoE) provides the connectivity and independent harmonization of a massive number of computing workstations, sensors, people, processes, entities, data, and machines that are linkup in a secure environment through the Network. Based on the current trend of this technology, it has demonstrated a high sense of transformation in our rational process of work, application of the machine, collaboration with people and/or machines, and extending to human consciousness. The scalability of loE has to lead to the unique invention as a solution to overcome the exceptional connectivity, miniaturization, interoperability, and utilisation of low energy efficiency. The loE platform is a secure environment that accomplishes many devices of all types and use a high classification set of rules in the authentication and authorisation of individual components online in other to prove their integrity and propagate through the global network, to the realisation and a solution to the digital world as applied to all sectors such as agriculture, payment, healthcare, smart grid, manufacturing, loT gateways, and industrial ecosystems.

\subsection{IoE Objectives}

i. The main objective is to provide computerization in the production area of the ecosystem.

ii. The transferred process from machine-to-machine, machine-to-people, and information technology to human technology.

iii. The collaboration of activities with people and machines to fit into the secure adaptation of the industrial framework.

\section{Literature Review}

I. Bandara \& F. Ioras (2016) explained that Internet of Everything (IoE) play an important role towards the connectivity of desktop computers, smartphone, tablet and laptop, and also allow the machines to become more intelligent and smarter in accessing data and work collaboratively with high-speed networks in the secure environment. W. Timothy, S. Architect (2014, Internet of Everything (loE) is built on the foundation of the Internet of Things (IOT) by adding more structures so that the networks become more intelligent that allow adaptation, convergence and visibility across the dissimilar systems in more reliable and valuable ways. IoE connects these devices into one single interconnected component allowing communication of everything with each other, which means that it is above devices communication. It is a philosophical technology's which future comprised of different types of appliances, devices, and items connected to the global Internet."

S. Wheeler, (2016) differentiates "IoE vs. IoT vs. M2M are explicitly linked with higher informative system globally and involve into the advancement of economic improvement, modernisation and new research that necessitated in the geometric progression of learning activities in a new dimension. It will allow people more access to the learning process regardless of their background. It includes: (1) to reduce the skills incongruity among what the labour force can do and what businesses need as indicators of success for loE adoption in higher instruction, (2) customization of curriculum to enable high/active engagement, interaction and attendance, and (3) access to right content and information and availability of materials on any device, at any time. loE filter, select and distinguish relevant information to the specific need of users. It is necessary to share and use knowledge so that the information is transformed into processes and products. D. J. Langley et al. (2018), improve on visualisation of how the loE may alter business models and the ways in which individuals and organizations create value in other to adopt a holistic approach.

A. Majeed et a (2016), formulated a secure loE architecture for cross-culture communication among organizations by concentrating on various technological wearable devices with a stronger security policy, through protocols, data format and data encryption structures to avoid data manipulation.

M. H. Miraz et al (2018), identify the 21 most important existing and forthcoming challenges as well as circumstances for the possible future expansion of their applications. 


\subsection{Internet of Everything (IOE)}

IoE allows communication and intercommunication of people, processes, data and things for the evaluability and relevancy of creating richer understandings, new competencies and unprecedented economic opportunity for individuals, businesses and countries. loE's consists of the four individual components which are shown in the figure below

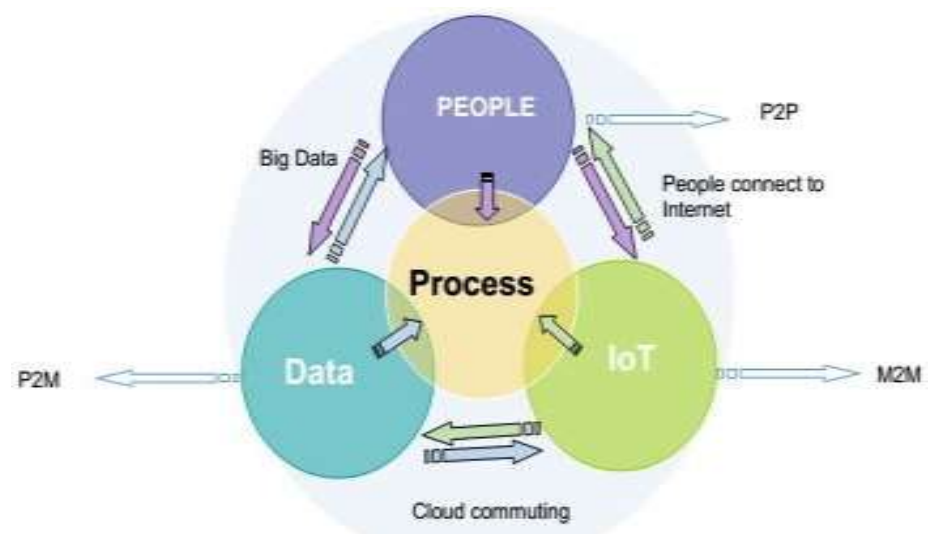

Figure 3: The What, Where, and How of the loE

1. People: The connection of PCs, laptops, smartphones, social networks and other devices through the Internet. But, with the development of IoE, people are connected in more relevant and valuable ways. For example, in a medical environment, a device inserted into the body of a patient will help to sense the medical condition of that patient and report to the doctor over a secure network connection. In this case, patients themselves act as internet slave that continuously monitors the information and transmitting the data to a storage system which is examined by the doctors in other to take corrective and timely action.

2. Data: This allows various devices to collect data and convey it over the internet to the storage point where it is quarried, analysed and handled in a more intelligent way. Therefore, the high-level information is sent back to computers, machines and people for further evaluation and decision making.

3. Things: It consists of peripheral, such as devices, sensors, and enterprises that are connected with each other through networked connections. The application of loE, sense more data, more valuable information that allows people and machines to make real-time decision making.

4. Process: This is pivot connectivity of people, data, and things in collaboration with other loE and delivers the information accordingly either to people or machines.

\subsection{The impact of IOE on businesses.}

The IoE has contributed three major impacts to the global economy, such as business strategies, human interaction with technology and technology adoption:

a. Business strategies: Living organizations adopt the IoE as a solution in the processing of their businesses benefitting, innovative and collaborative ideology when connected with a global network.

b. Human interaction with technology: With the emergence of loE technology, there is an increased level of human interaction with technology, as people-to-people(P2P), machine-to-machine (M2M), people-to-machine (P2M) and machine-to-people (M2P) communication provides a good interface in term of collaboration between people and things.

c. Technology adoption: IoE relies on network connectivity to deploy the solutions that offer programmability, improved flexibility and enhanced policy management by every sector.

\subsection{Features and Applications of IoE.}

The various features are

i. Single addressability of entity

ii. Exclusive position and identification of object within a network

iii. The human Interaction and interface of people-to-people (P2P), machine-to-machine (M2M), people-to-machine (P2M), and machine-to-people (M2P) that lead to collaborative and efficiency in the global network domain. 
iv. The superimposition of intelligence, analytical hypothesis, security, and management acquisitions.

v. The urgent need for information in real-time becomes the norm.

Above all, loE can be applied in the figure shown below

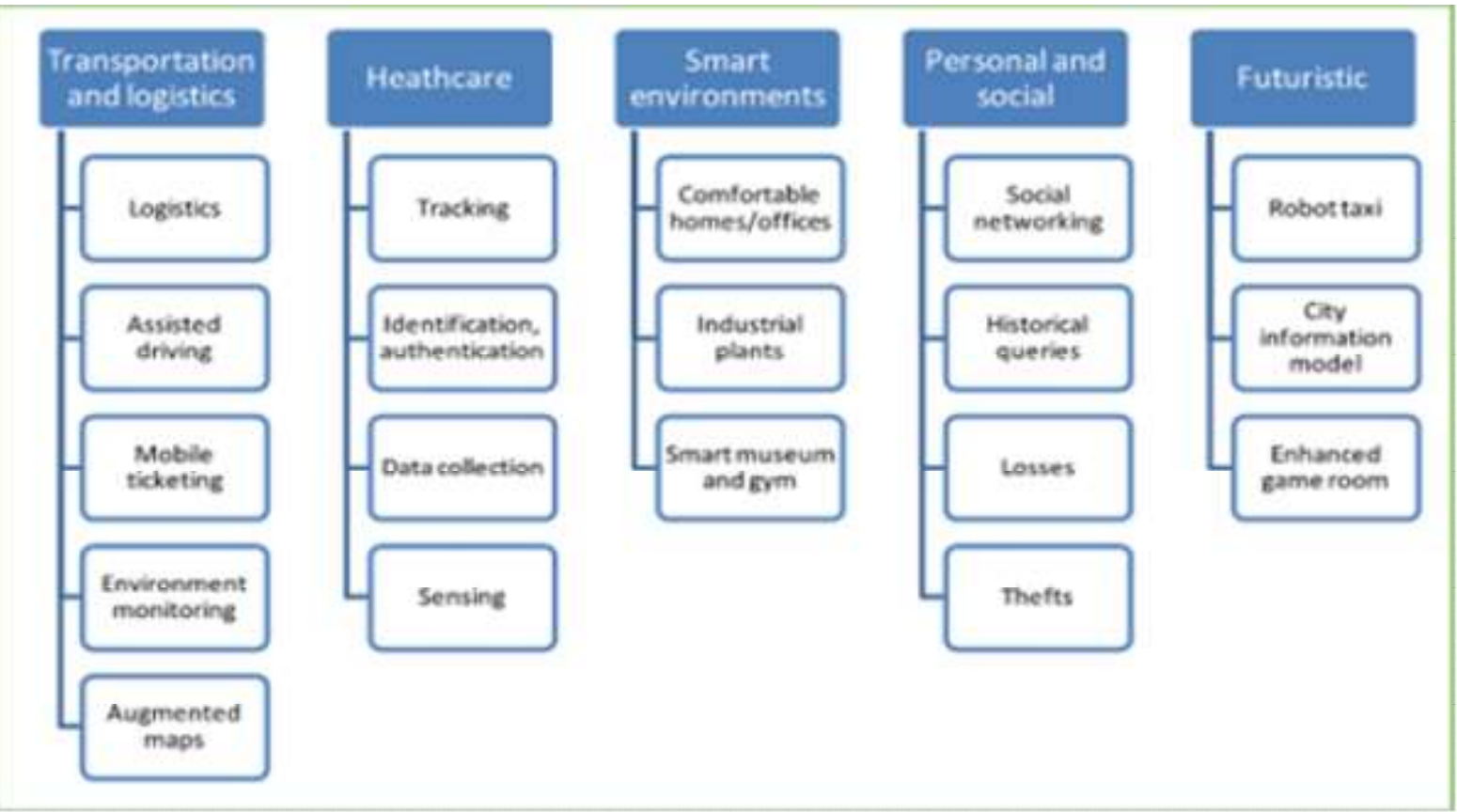

\subsection{Future of $10 E$}

loE allows Living and non-living things to communicate with the help of sensors and Internet availability as a backbone connector. It amplifies the regulation and productivity of things to the comfort of our zone, for example, in the area of the medical environment, it allows us to plant a sensor on a deaf and drum person as a means of communication between the disabled person and the Doctor. The doctor needs to query the deaf person through the Sensor in other to ascertain the course of the problem for easy drug recommendation and treatment.

The economic significance created by smart devices will actually increase from $\$ 10$ trillion to $\$ 20$ trillion in the next five years. Different devices of loE products, services, and suppliers will progress geometrically in terms of revenue generation to exceed $\$ 300$ billion in 2022. There are needs for us to adapt to the changing world of loE devices and Technological innovations towards the paradigm of demand and supply.

\subsection{Challenges in IoE}

The integration of loE devices with various wireless networks, spectrum resources, security, low data rate, a large volume of links, power, automatic deployment and maintenance is a big challenge to loE. The multitude of endpoint connectors and sensors that collect, analyse and transmit data on a massive scale automatically, the volume and velocity of this data make it cumbersome to bring together at the speed of light. This means that what was recognised as real-time technology will now be considered online technology. Another challenge is the high intensity of video surveillance cameras combined with facial recognition data from age, gender and socioeconomic aspects. However, not all the data from these devices need to be stored rather, it can be used for that moment to create interactive engagements with the end-users.

Another important challenge is security, starting from first points to endpoints of device connectivity. It is necessary for us to design the various network-centric security approaches due to the wireless connectivity of static and dynamic devices

\section{Conclusions}

loE technology has the capability to transform the world in the area of interconnectivity, big data, artificial intelligence and semantic operability. Organizations need to play vibrant roles to influence the way of loE that will energies our societies. The loE is expected to open up new prospects and pose new challenges to different disciplines such as computer science, informatics science, psychology, law, economics and ethics is needed to better understand and influence future developments in the loE. Internet of Everything (loE) changes only one thing, which is "everything. 


\section{References}

[1] Bandara, I., \& loras, F. (2016). The evolving challenges of internet of everything: enhancing student performance and employability in higher education. INTED2016 10th annual International Technology, Education and Development, 652-658.

[2] Borgia, E. (2014). The Internet of Things vision: Key features, applications and open issues. Computer Communications, 54, 1-31.

[3] Gubbi, J., Buyya, R., Marusic, S., \& Palaniswami, M. (2013). Internet of Things (IoT): A vision, architectural elements, and future directions. Future generation computer systems, 29(7), 1645-1660.

[4] Langley, D. J., van Doorn, J., Ng, I. C., Stieglitz, S., Lazovik, A., \& Boonstra, A. (2021). The Internet of Everything: Smart things and their impact on business models. Journal of Business Research, 122, 853-863.

[5] Majeed, A., Bhana, R., Haq, A., Kyaruzi, I., \& Williams, M. (2016). Devising a secure architecture of internet of everything (loE) to avoid the data exploitation in cross culture communications. International Journal of Advanced Computer Science and Applications (IJACSA), 7(4), 328333.

[6] Miraz, M., Ali, M., Excell, P., \& Picking, R. Internet of nano-things, things and everything: future growth trends. Future Internet 10 (8), 68 (2018).

[7] Rajiv, S. (2021). Gurjeet (2021). Future of Internet of Everything (IOE). IRJCS:: International Research Journal of Computer Science, Volume VIII, 84-92.

[8] Wheeler, S. (2016). loE vs. IoT vs. M2M: What's the Difference and Does It Matter. loT Tech Expo. Pobrane z: http://www. iottechexpo. com/2016/01/m2m/ioe-vs-iot-vs-m2m-whats--the-difference-and-does-it-matter/(29.04. 2018). 